Supporting information for

\title{
FLUORECENT STAINING FOR STUDY OF EXTRACELLULAR POLYMERIC SUBSTANCES IN MEMBRANE BIOFOULING LAYERS
}

\author{
Ming-Yuan Chen ${ }^{1}$, Duu-Jong Lee ${ }^{1, *}$, Z. Yang ${ }^{2}$, X.F. Peng ${ }^{2}$, J. Y. Lai ${ }^{3}$ \\ ${ }^{1}$ Chemical Engineering Department, National Taiwan University, Taipei, 106 Taiwan \\ ${ }^{2}$ Department of Thermal Engineering, Tsinghua University, Beijing, 100084, China \\ ${ }^{3}$ Department of Chemical Engineering, R\&D Center of Membrane Technology, Chung \\ Yuan Christian University, Chungli, Taiwan 32023 \\ *Corresponding author: (voice) +886-2-2362-5632 (Email) djlee@ntu.edu.tw
}

Published by Environmental Science \& Technology

The document was prepared on May, 29, 2006/8/22

This 4-page Supporting Information contains three figures and no table. 
Figure S1 presents a CLSM image of the microtome of the fouling layer viewed from its side, combined with a $15-\mu \mathrm{m}$ thick membrane body underneath.

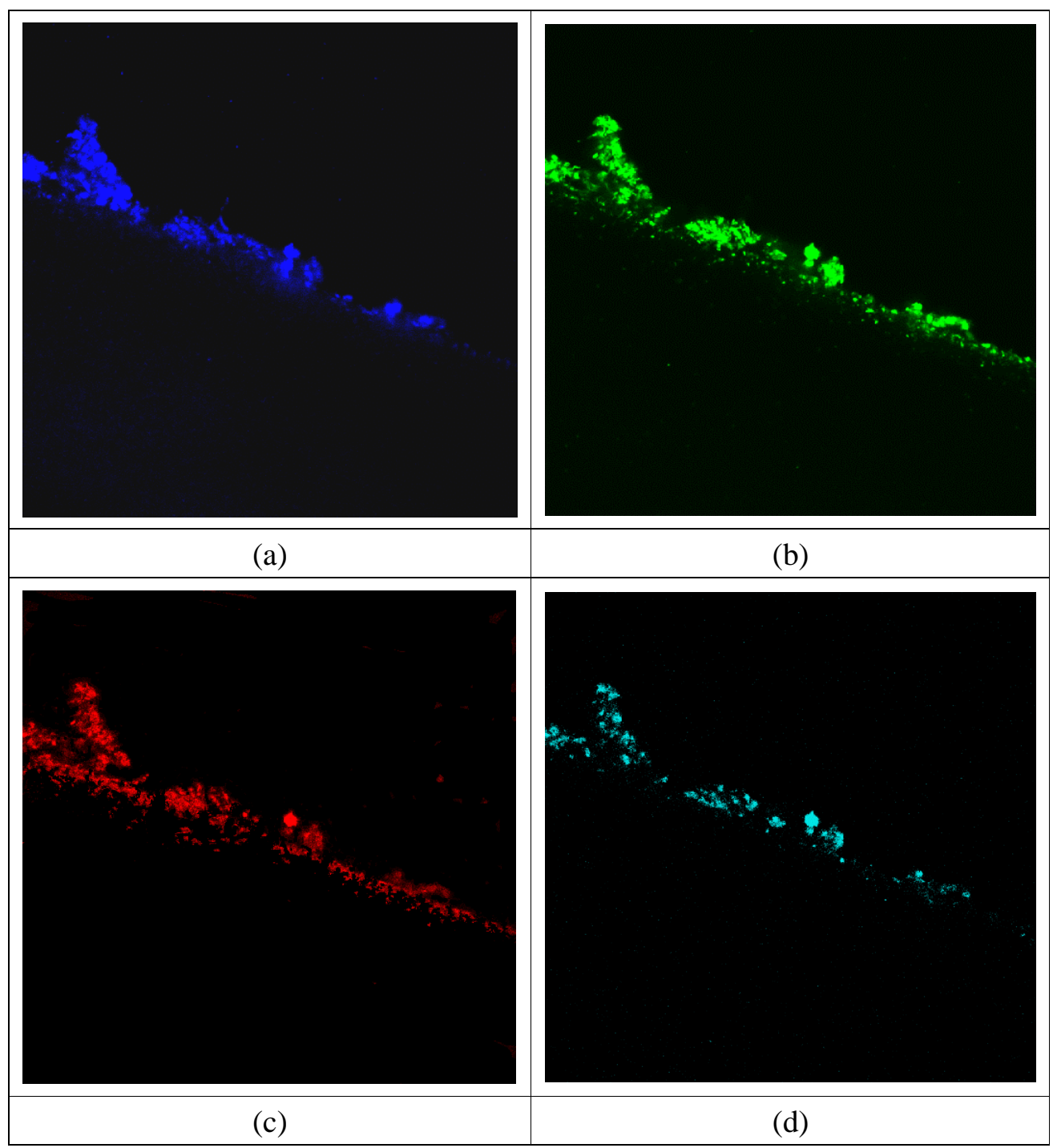

Figure S1. CLSM images of microtome of fouling layer cutting at the center of the filter membrane. (a) CLSM image of $\beta$-D-glucopyranose polysaccharides (calcofluor white); (b) CLSM image of proteins (FITC); (c) CLSM image of nucleic acids (SYTO 63); (d) CLSM image of $\alpha$-D-glucopyranose polysaccharides (Con A).

Figure S2 in presents an image combining Figs. S1a-S1d. Numerical values indicate the thickness of fouling layer counting from the membrane surface. This cross-sectional view suggests that the fouling layer has a thickness of $6.8-17.8 \mu \mathrm{m}$, and a very non-uniform spatial distribution. 




Figure S2. Quadruple staining results combining images of Figs. 1a-1d. Numerical values indicate the thickness of fouling layer counting from the membrane surface. 
Figure S3a presents an image combining Figs. 2a-d of a CLSM scanned image at 10 $\mu \mathrm{m}$ from the membrane surface. Fig. S3b is the bileveled image of Fig. S3a. The threshold value was determined using Otsu's scheme.

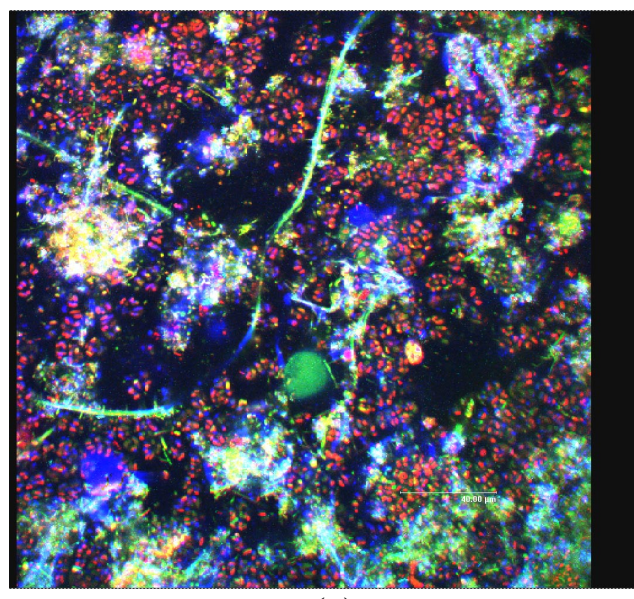

(a)

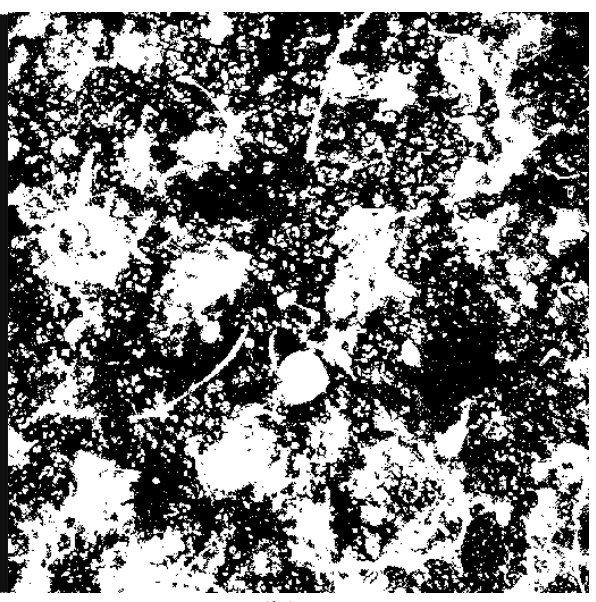

(b)

Figure S3. Images of fouling layer scanning at $10 \mu \mathrm{m}$ above membrane surface. (a) Quadruple staining results combining images of Figs. 2a-2d. (b) Bileveled image using Otsu's method. 